\title{
A General Iteration Formula of VIM for Fractional Heat- and Wave-Like Equations
}

\author{
Fukang Yin, Junqiang Song, and Xiaoqun Cao \\ Department of Software, College of Computer, National University of Defense Technology, Changsha, Hunan 410073, China \\ Correspondence should be addressed to Fukang Yin; yinfukang@nudt.edu.cn
}

Received 24 December 2012; Accepted 2 March 2013

Academic Editor: Zhongxiao Jia

Copyright (C) 2013 Fukang Yin et al. This is an open access article distributed under the Creative Commons Attribution License, which permits unrestricted use, distribution, and reproduction in any medium, provided the original work is properly cited.

\begin{abstract}
A general iteration formula of variational iteration method (VIM) for fractional heat- and wave-like equations with variable coefficients is derived. Compared with previous work, the Lagrange multiplier of the method is identified in a more accurate way by employing Laplace's transform of fractional order. The fractional derivative is considered in Jumarie's sense. The results are more accurate than those obtained by classical VIM and the same as ADM. It is shown that the proposed iteration formula is efficient and simple.
\end{abstract}

\section{Introduction}

Fractional differential equations (FDEs) have been proved to be a valuable tool in the modelling of many phenomena in the fields of applied sciences. This is because of the fact that fractional derivatives provide an excellent instrument to describe the memory and hereditary properties of various materials and processes. With the increasing applications of FDEs, considerable attentions have been paid to provide efficient methods for finding the exact and numerical solutions of FDEs. Recently, some approximate methods such as Adomian's decomposition method [1-5], homotopy perturbation method [6-10], variational iteration method [11-27], and homotopy analysis method [28, 29] are given to find an analytical approximation to FDEs.

The variational iteration method (VIM) was first proposed by He et al. [11-15] and has been shown to be efficient for handling nonlinear problems. Thus, there has been a great deal of interest in FDEs by using the VIM [17-27]. For VIM, the key points are the construction of correct function and the identification of the Lagrange multiplier. However, the previous work either avoids the term of fractional derivative and handles them as a restricted variation or identifies the Lagrange multipliers by an approximate method, resulting in a poor convergence and inaccuracy. So, it is urgent to find a new method which can identify the Lagrange multiplier in a more accurate way.
In this paper, we will use a correct function described in [27] and give a new method to evaluate the Lagrange multipliers by employing Laplace's transform of fractional order. Then, we develop a new framework of VIM for the three-dimensional fractional heat- and wave-like equations of the form [3]:

$$
\begin{gathered}
\frac{\partial^{\alpha} T}{\partial t^{\alpha}}=f(x, y, z) T_{x x}+g(x, y, z) T_{y y}+h(x, y, z) T_{z z} \\
0<x<a, 0<y<b, 0<z<c, n<\alpha<n+1, t>0,
\end{gathered}
$$

subject to the boundary conditions

$$
\begin{aligned}
T(0, y, z, t) & =f_{1}(y, z, t), & T_{x}(a, y, z, t) & =f_{2}(y, z, t), \\
T(x, 0, z, t) & =g_{1}(x, z, t), & T_{y}(x, b, z, t) & =g_{2}(x, z, t), \\
T(x, y, 0, t) & =h_{1}(x, y, t), & T_{z}(x, y, c, t) & =h_{2}(x, y, t),
\end{aligned}
$$

and the initial conditions

$$
T(x, y, z, 0)=\psi(x, y, z), \quad T_{t}(x, y, z, 0)=\eta(x, y, z),
$$

where $\alpha$ is a parameter describing the fractional derivative. In the case of $0<a \leq 1$, (1) reduces to the fractional heat-like 
equation with variable coefficients and to the fractional wave-like equation that models anomalous diffusive and subdiffusive systems in the case of $1<a \leq 2$. The approximate solutions of (1) have been studied by using the ADM [3], VIM [17], FVIM [23], and modified VIM [24].

The remainder of the paper is organized as follows. In Section 2, we describe some necessary preliminaries of the fractional calculus and the Laplace transform for our subsequent development. Section 3 is devoted to the derivation of general iteration formula for the fractional heatand wave-like equations. In Section 4, four examples are given to demonstrate our conclusions. Finally, a brief summary is presented.

\section{Preliminaries}

In this section, we cover some preliminaries. First, we list some basic definitions about fractional calculus. Second, the Laplace transforms of fractional integral and derivative are described. For more details, see [30-39].

\subsection{Fractional Calculus}

Definition 1. A real function $h(t), t>0$, is said to be in the space $C_{\mu}, \mu \in R$, if there exist a real number $p>\mu$, such that $h(t)=t^{p} h_{1}(t)$, where $h_{1}(t) \in C(0, \infty)$, and it is said to be in the space $C_{\mu}^{n}$ if and only if $h^{(n)} \in C_{\mu}, n \in N$.

Definition 2. The Riemann-Liouville fractional integral operator $\left(J^{\alpha}\right)$ of order $\alpha \geq 0$, of a function $f \in C_{\mu}, \mu \geq-1$, is defined as

$$
\begin{gathered}
J^{\alpha} f(t)=\frac{1}{\Gamma(\alpha)} \int_{0}^{t}(t-\tau)^{\alpha-1} f(\tau) d \tau, \quad t>0, \\
J^{0} f(t)=f(t),
\end{gathered}
$$

when $0<\alpha \leq 1, J^{\alpha} f(t)=(1 / \Gamma(\alpha)) \int_{0}^{t}(t-\tau)^{\alpha-1} f(\tau) d \tau=$ $(1 / \Gamma(1+\alpha)) \int_{0}^{t} f(\tau)(d \tau)^{\alpha}$.

If we denote the Riemann-Liouville fractional derivative by $D^{\alpha}$, then the next equation define the Riemann-Liouville fractional derivative of order $m$

$$
D^{\alpha} f(x)=\frac{d^{m}}{d x^{m}}\left(J^{m-\alpha} f(x)\right)
$$

where $m-1<\alpha \leq m, m \in N$.

Jumarie (see [31-37]) proposed a simple alternative definition to the Riemann-Liouville derivative. Since his modified Riemann-Liouville derivative is defined for arbitrary continuous (nondifferentiable) functions and the fractional derivative of a constant is equal to zero, it has the advantages of both the standard Riemann-Liouville and the Caputo fractional derivatives. Now, we present some notations, definitions, and preliminary facts of the modified RiemannLiouville derivative which will be used later in this work.
Definition 3. Jumarie's fractional derivative is a modified Riemann-Liouville derivative defined by the expression [37, 38]

$$
f^{(\alpha)}(x):=\frac{1}{\Gamma(-\alpha)} \int_{0}^{x}(x-\xi)^{-\alpha-1} f(\xi) d \xi, \quad \alpha<0 .
$$

For positive $\alpha$, one will set

$$
\begin{aligned}
f^{(\alpha)}(x):= & \left(f^{(\alpha-1)}(x)\right)^{\prime} \\
= & \frac{1}{\Gamma(1-\alpha)} \frac{d}{d x} \\
& \times \int_{0}^{x}(x-\xi)^{-\alpha}(f(\xi)-f(0)) d \xi, \quad 0<\alpha<0, \\
f^{(\alpha)}(x) & :=\left(f^{(\alpha-n)}(x)\right)^{(n)}, \quad n \leq \alpha<n+1, n \geq 1 .
\end{aligned}
$$

In addition, we want to give some properties of Jumarie's fractional derivative.

Theorem 4 (the fractional Leibniz product rule [36]). If $f$ and $g$ are two continuous functions on $[0,1]$, then

$$
(f(x) g(x))^{\alpha}=(f(x))^{\alpha} g(x)+f(x)(g(x))^{\alpha} .
$$

Theorem 5 (the fractional Barrow's formula [37]). For a continuous function $f$, one has

$$
\int_{0}^{x} f^{(\alpha)}(t)(d t)^{(\alpha)}=\alpha !(f(x)-f(0))
$$

where $\alpha !=\Gamma(1+\alpha)$.

From Theorems 4 and 5, the formula of integration by parts is given as

$$
\begin{aligned}
& \int_{0}^{1} u^{(\alpha)}(x) v(x)(d x)^{(\alpha)} \\
& \quad=\int_{0}^{1}(u(x) v(x))^{(\alpha)}(d x)^{(\alpha)}-\int_{0}^{1} u(x) v^{(\alpha)}(x)(d x)^{(\alpha)} \\
& \quad=\alpha ![u(x) v(x)]_{0}^{1}-\int_{0}^{1} u(x) v^{(\alpha)}(x)(d x)^{(\alpha)} .
\end{aligned}
$$

Definition 6. Fractional derivative of compounded functions $[37,38]$ is defined as

$$
d^{\alpha} f \cong \Gamma(1+\alpha) d f, \quad 0<\alpha<1 .
$$

Definition 7. The integral with respect to $(d x)^{\alpha}[37,38]$ is defined as the solution of the fractional differential equation

$$
d y \cong f(x)(d x)^{\alpha}, \quad x \geq 0, y(0)=0,0<\alpha<1 .
$$

Lemma 8. Let $f(x)$ denote a continuous function [37, 38], then the solution $y(x), y(0)=0$ of $(8)$ is defined by the equality

$$
y=\int_{0}^{x} f(\xi)(d \xi)^{\alpha}=\alpha \int_{0}^{x}(x-\xi)^{\alpha} f(\xi) d \xi, \quad 0<\alpha<1
$$


Lemma 9. Let $n-1<\alpha \leq n, n \in N, t>0, h \in C_{\mu}^{n}, \mu \geq-1$, then

$$
\left(J^{\alpha} D^{\alpha}\right) h(t)=h(t)-\sum_{k=0}^{n-1} h^{(k)}\left(0^{+}\right) \frac{t^{k}}{k !} .
$$

\subsection{The Laplace Transform}

Definition 10. The Laplace transform $L\{f(x)\}:=F(s), s \in C$, of a $R \rightarrow C$ function $f(x)$ is defined by the integral

$$
L\{f(x)\}:=: F(s):=\int_{0}^{\infty} e^{s x} f(x) d x .
$$

Lemma 11. The Laplace transform of the fractional derivative (the Riemann-Liouville derivative) is

$$
L\left\{D_{x}^{\alpha} f(x) ; s\right\}=s^{\alpha} F(s)-\sum_{k=1}^{n} s^{k-1} D^{\alpha-k} f(0+) .
$$

Definition 12. The inverse Laplace transform is defined by the complex integral

$$
f(t)=L^{-1}\{F(s)\}=\frac{1}{2 \pi i} \lim _{T \rightarrow \infty} \int_{\rho-i T}^{\rho+i T} e^{s t} F(s) d s,
$$

where the integration is done along the vertically $\operatorname{Re}(s)=\rho$ in the complex plane such that $\rho$ is greater than the real part of all singularities of $F(s)$. This ensures that the contour path is in the region of convergence. If all singularities are in the left half-plane, or $F(s)$ is a smooth function on $-\infty<$ $\operatorname{Re}(s)<\infty$ (i.e., no singularities), then $\rho$ can be set to zero and the above inverse integral formula becomes identical to the inverse Fourier transform.

Now, we will introduce the definition of the fractional Laplace transform derived by Jumarie [38] for the first time, and some results of the fractional Laplace transform are also presented.

Definition 13. Let $f(x)$ denote a function which vanishes for negative values of $x$. Its Laplace's transform $L_{\alpha}\{f(x)\}$ of order $\alpha$ (or its $\alpha$ th fractional Laplace's transform) is defined by the following expression, when it is finite:

$$
\begin{array}{r}
L_{\alpha}\{f(x)\}:=: F_{\alpha}(s)=\int_{0}^{\infty} E_{\alpha}\left(-s^{\alpha} x^{\alpha}\right) f(x)(d x)^{\alpha}, \\
0<\alpha \leq 1,
\end{array}
$$

where $s \in C$ and $E_{\alpha}(u)$ is the Mittag-Leffler function $\sum_{k=0}^{\infty}\left(u^{k} / \Gamma(1+k \alpha)\right)$.

Lemma 14. If one defines the convolution of order $\alpha$ of the two functions $f(x)$ by the expression

$$
(f(x) * g(x))_{\alpha}:=\int_{0}^{x} f(x-u) g(u)(d u)^{\alpha}, \quad 0<\alpha \leq 1 .
$$

Then, one has the equality

$$
L_{\alpha}\left\{(f(x) * g(x))_{\alpha}\right\}=L_{\alpha}\{f(x)\} L_{\alpha}\{g(x)\} .
$$

Corollary 15. Given the Laplace transform that one has the inversion formula

$$
f(x)=\frac{1}{\left(M_{\alpha}\right)} \int_{-i \infty}^{+i \infty} E_{\alpha}\left(s^{\alpha} x^{\alpha}\right) F_{\alpha}(s)(d s)^{\alpha}, \quad 0<\alpha \leq 1,
$$

where $M_{\alpha}$ is the period of the complex-valued Mittag-Leffler function defined by the equality $E_{\alpha}\left(i\left(M_{\alpha}\right)^{\alpha}\right)=1$.

Lemma 16. The fractional Laplace transform of the fractional derivative (the modified Riemann-Liouville derivative) is

$$
L_{\alpha}\left\{f^{(\alpha)}(x)\right\}=s^{\alpha} L_{\alpha}\{f(x)\}-\Gamma(1+\alpha) f(0), \quad 0<\alpha \leq 1 .
$$

Some properties of the fractional Laplace transform are given as follows [38]:

$$
\begin{gathered}
L_{\alpha}\left\{x^{\alpha} f(x)\right\}=-D_{s}^{\alpha} L\{f(x)\}, \\
L_{\alpha}\{f(a x)\}_{s}=\left(\frac{1}{a}\right)^{\alpha} L_{\alpha}\{f(x)\}_{s / \alpha}, \\
L_{\alpha}\{f(x-b)\}=E_{\alpha}\left(-s^{\alpha} b^{\alpha}\right) L_{\alpha}\{f(x)\}, \\
L_{\alpha}\left\{E_{\alpha}\left(-c^{\alpha} x^{\alpha}\right) f(x)\right\}_{s}=L_{\alpha}\{f(x)\}_{s+c}, \\
L_{\alpha}\left\{-x^{\alpha} f(x)\right\}=D_{s}^{\alpha} L_{\alpha}\{f(x)\}, \\
L_{\alpha}\left\{\int_{0}^{x} f(u)(d u)^{\alpha}\right\}=\Gamma^{-1}(1+\alpha) s^{-\alpha} L_{\alpha}\{f(x)\} .
\end{gathered}
$$

\section{Fractional Variational Iteration Method}

In order to illustrate the solution procedure of the variational iteration method, we consider the following fractional differential equation:

$$
\begin{array}{r}
D_{t}^{\alpha} u(x, y, t)+N u(x, y, t)+R u(x, y, t)=g(x, y, t), \\
t>0, m-1<\alpha \leq m,
\end{array}
$$

where $R$ is the linear operator, $N$ is the nonlinear operator, and $D_{t}^{\alpha}$ is the modified Rieman-Liouville derivative of order $\alpha$.

Subject to the initial condition,

$$
u^{(k)}(x, y, 0)=c_{k}(x, y), \quad k=0,1, \ldots, m
$$

Let $u_{k}(t)=u_{k}(x, y, t), g_{k}(t)=g_{k}(x, y, t)$. 
Before our solution, we will describe the fractional variational iteration method described in $[23,25,26]$, which construct a correct function for (24) as

$$
\begin{array}{r}
u_{k+1}(t)=u_{k}(t) \\
+J_{\tau}^{\alpha}\left[\lambda ( t , \tau ) \left(D_{\tau}^{\alpha} u_{k}(\tau)+N \widetilde{u}_{k}(\tau)\right.\right. \\
\left.\left.+R u_{k}(\tau)-g(\tau)\right)\right] \\
=u_{k}(t)+\frac{1}{\Gamma(\alpha)} \int_{0}^{t}(t-\tau)^{\alpha-1} \lambda(t, \tau) \\
\times\left(D_{\tau}^{\alpha} u_{k}(\tau)+N \widetilde{u}_{k}(\tau)\right. \\
\left.+R u_{k}(\tau)-g(\tau)\right) d \tau .
\end{array}
$$

Then, by using Lemma 8 proposed by Jumarie $[37,38]$ for the first time, one gets a correction function

$$
\begin{aligned}
u_{k+1}(t)= & u_{k}(t)+\frac{1}{\Gamma(1+\alpha)} \\
& \times \int_{0}^{t} \lambda(t, \tau)\left(D_{\tau}^{\alpha} u_{k}(\tau)+N \tilde{u}_{k}(\tau)\right. \\
& \left.\quad+R u_{k}(\tau)-g(\tau)\right)(d \tau)^{\alpha} .
\end{aligned}
$$

But, unfortunately, (27) holds true only when $0<\alpha \leq 1$. In the case of $m-1<\alpha \leq m, m=2,3,4, \ldots$, some modifications must be made. For example, replacing the fractional order $\alpha$ $(m-1<\alpha \leq m)$ by the order $m \alpha(0<\alpha \leq 1)$, whether there is a general iteration formula for (24). Certainly, there is. In the remainder section, we will use two methods to derive a general iteration formula of VIM for (24). Unlike the previous work, which calculates the Lagrange multiplier by some approximate methods, we will use a more accurate way by employing the properties of Laplace's transform.

3.1. The Laplace Transform Method. According to VIM [27], we can construct a correction functional as follows:

$$
\begin{array}{r}
u_{k+1}(t)=u_{k}(t)+J_{\tau}^{\beta}\left[\lambda ( t , \tau ) \left(D_{\tau}^{\alpha} u_{k}(\tau)+N \tilde{u}_{k}(\tau)\right.\right. \\
\left.\left.+R u_{k}(\tau)-g(\tau)\right)\right] \\
=u_{k}(t)+\frac{1}{\Gamma(\beta)} \int_{0}^{t}(t-\tau)^{\beta-1} \lambda(t, \tau) \\
\times\left(D_{\tau}^{\alpha} u_{k}(\tau)+N \tilde{u}_{k}(\tau)\right. \\
\left.+R u_{k}(\tau)-g(\tau)\right) d \tau,
\end{array}
$$

where $J_{\tau}^{\beta}$ is the Riemann-Liouville fractional integral operator of order $\beta=\alpha$-floor $(\alpha)$, that is, $\beta=\alpha+1-m$, with respect to the variable $t$, and $\lambda$ is a general Lagrange multiplier, and $\tilde{u}_{k}(\tau)$ is a restricted variation, that is, $\delta \widetilde{u}(\tau)=0$.
By taking the Laplace transform on the both sides of (28), we have

$$
\begin{aligned}
& L\left\{u_{k+1}(t)\right\} \\
& =L\left\{u_{k}(t)\right\} \\
& \quad+L\left\{\frac{1}{\Gamma(\beta)} \int_{0}^{t}(t-\tau)^{\beta-1} \lambda(t, \tau)\right. \\
& \quad \times\left(D_{\tau}^{\alpha} u_{k}(\tau)+N \tilde{u}_{k}(\tau)\right. \\
& \left.\left.\quad+R u_{k}(\tau)-g(\tau)\right) d \tau\right\},
\end{aligned}
$$

where $L$ is the operator of the Laplace transform.

$$
\begin{aligned}
L\left\{u_{k+1}(t)\right\}= & L\left\{u_{k}(t)\right\}+\frac{1}{\Gamma(\beta)} L\left\{(t-\tau)^{\beta-1} \lambda(t, \tau)\right\} \\
& \times L\left\{D_{\tau}^{\alpha} u_{k}(\tau)+N \tilde{u}_{k}(\tau)+R u_{k}(\tau)-g(\tau)\right\} .
\end{aligned}
$$

From the Lemma 11, we get

$$
\begin{array}{r}
L\left\{u_{k+1}(t)\right\}=L\left\{u_{k}(t)\right\}+\frac{1}{\Gamma(\beta)} L\left\{(t-\tau)^{\beta-1} \lambda(t, \tau)\right\} \\
\times L\left\{s^{\alpha} L\left\{u_{k}(\tau)\right\}-\sum_{k=1}^{n} s^{k-1} D^{\alpha-k} u_{k}(0)\right. \\
\left.+N\left[\widetilde{u}_{k}(\tau)\right]+R\left[u_{k}(\tau)\right]-g(\tau)\right\} .
\end{array}
$$

Taking the variation derivative $\delta$ on the both sides of (31), we can derive

$$
\begin{aligned}
L\left\{u_{k+1}(t)\right\}= & L\left\{u_{k}(t)\right\} \\
& +\frac{1}{\Gamma(\beta)} L\left\{(t-\tau)^{\beta-1} \lambda(t, \tau)\right\} \\
& \times \delta\left[s^{\alpha} L\left\{u_{k}(\tau)\right\}+L\left\{R\left[u_{k}(\tau)\right]\right\}\right] .
\end{aligned}
$$

Without loss of generality, assuming that $\delta R\left[u_{k}(\tau)\right]=0$, then we have

$$
\delta L\left\{u_{k}(t)\right\}: 1+\frac{1}{\Gamma(\beta)} s^{\alpha} L\left\{(t-\tau)^{\beta-1} \lambda(t, \tau)\right\}=0 .
$$

Set the coefficient of $\delta L\left\{u_{k}(t)\right\}$ to zeros, we obtain

$$
L\left\{(t-\tau)^{\beta-1} \lambda(t, \tau)\right\}=-\Gamma(\beta) s^{-\alpha} .
$$

By employing the inverse Laplace transform, we have

$$
(t-\tau)^{\beta-1} \lambda(t, \tau)=-\frac{(t-\tau)^{\alpha-1} \Gamma(\beta)}{\Gamma(\alpha)} .
$$

Substituting (35) into (28), we get the iteration formula as follows:

$$
\begin{aligned}
u_{k+1}(t)= & u_{k}(t) \\
& -J_{\tau}^{\alpha}\left\{\left(D_{t}^{\alpha} u_{k}(\tau)+N u_{k}(\tau)+R u_{k}(\tau)-g(\tau)\right)\right\} .
\end{aligned}
$$


3.2. The Fractional Laplace Transform Method. In order to illustrate our method, we replace the fractional order $\alpha(m-$ $1<\alpha \leq m)$ by the order $m \alpha(0<\alpha \leq 1)$. According to VIM [27], we can construct a correction function as follows:

$$
\begin{aligned}
u_{k+1}(t)= & u_{k}(t) \\
& +J_{\tau}^{\alpha}\left[\lambda ( t , \tau ) \left(D_{\tau}^{m \alpha} u_{k}(\tau)+N \widetilde{u}_{k}(\tau)\right.\right. \\
& \left.\left.+R u_{k}(\tau)-g(\tau)\right)\right] .
\end{aligned}
$$

Taking the fractional Laplace transform on the both sides of (37), we have

$$
\begin{aligned}
L_{\alpha}\left\{u_{k+1}(t)\right\}= & L_{\alpha}\left\{u_{k}(t)\right\} \\
& +L_{\alpha}\left\{J _ { \tau } ^ { \alpha } \left[\lambda ( t , \tau ) \left(D_{\tau}^{m \alpha} u_{k}(\tau)+N \widetilde{u}_{k}(\tau)\right.\right.\right. \\
& \left.\left.\left.+R u_{k}(\tau)-g(\tau)\right)\right]\right\},
\end{aligned}
$$

where $L_{\alpha}$ is the fractional Laplace transform of order $\alpha$.

By assuming that $\lambda(t, \tau)$ has the form as $\lambda(t, \tau)=\lambda(t-\tau)$ and using (10), then we get

$$
\begin{aligned}
J_{\tau}^{\alpha} \lambda & \left(D_{\tau}^{m \alpha} u_{k}(\tau)+N \widetilde{u}_{k}(\tau)+R u_{k}(\tau)-g(\tau)\right) \\
= & \frac{1}{\Gamma(1+\alpha)} \\
& \times\left(\lambda(t, \tau) *\left(D_{\tau}^{m \alpha} u_{k}(\tau)+N \tilde{u}_{k}(\tau)+R u_{k}(\tau)-g(\tau)\right)\right)_{\alpha} .
\end{aligned}
$$

From (20), we have

$$
\begin{aligned}
L_{\alpha} & \left\{J_{\tau}^{\alpha} \lambda\left(D_{\tau}^{m \alpha} u_{k}(\tau)+N \widetilde{u}_{k}(\tau)+R u_{k}(\tau)-g(\tau)\right)\right\} \\
= & \frac{1}{\Gamma(1+\alpha)} L_{\alpha}\{\lambda(t, \tau)\} \\
& \times L_{\alpha}\left\{D_{\tau}^{m \alpha} u_{k}(\tau)+N \widetilde{u}_{k}(\tau)+R u_{k}(\tau)-g(\tau)\right\} .
\end{aligned}
$$

Substituting (40) into (38) and then taking the variation derivative $\delta$ on the both sides of (35), we can derive

$$
\begin{aligned}
L_{\alpha}\left\{u_{k+1}(t)\right\}= & L_{\alpha}\left\{u_{k}(t)\right\} \\
& +\frac{1}{\Gamma(1+\alpha)} L_{\alpha}\{\lambda(t-\tau)\} \\
& \times \delta\left[L\left\{D_{\tau}^{m \alpha} u_{k}(\tau)+R u_{k}(\tau)\right\}\right] .
\end{aligned}
$$

Without loss of generality, assuming that $\delta\left[R u_{k}(\tau)\right]=0$, then we have

$$
\delta L_{\alpha}\left\{u_{k}(t)\right\}: 1+\frac{1}{\Gamma(1+\alpha)} L_{\alpha}\{\lambda(t-\tau)\} s^{m \alpha}=0 .
$$

Set the coefficient of $\delta L_{\alpha}\left\{u_{k}(t)\right\}$ to zeros, we have

$$
L_{\alpha}\{\lambda(t-\tau)\}=-\frac{\Gamma(1+\alpha)}{s^{m \alpha}} .
$$

By employing the inverse fractional Laplace transform, we have

$$
\lambda(t, \tau)=-\frac{\Gamma(1+\alpha)}{\Gamma(1+(m-1) \alpha)}(t-\tau)^{(m-1) \alpha} .
$$

Substituting (40) into (26), we get the iteration formula as follows:

$$
\begin{aligned}
u_{k+1}(t)= & u_{k}(t) \\
& +J_{\tau}^{\alpha}\left\{-\frac{\Gamma(1+\alpha)}{\Gamma(1+(m-1) \alpha)}(t-\tau)^{(m-1) \alpha}\right. \\
& \left.\times\left(D_{\tau}^{m \alpha} u_{k}(\tau)+N u_{k}(\tau)+R u_{k}(\tau)-g(\tau)\right)\right\} \\
= & u_{k}(t) \\
& \quad-J_{\tau}^{m \alpha}\left\{D_{\tau}^{m \alpha} u_{k}(\tau)+N u_{k}(\tau)+R u_{k}(\tau)-g(\tau)\right\} .
\end{aligned}
$$

After replacing the fractional order $m \alpha(0<\alpha \leq 1)$ by the order $\alpha(m-1<\alpha \leq m)$, we could find that (36) and (45) are the same. So, we could get a general iteration formula for (1) as follows:

$$
\begin{aligned}
& T_{k+1}(t) \\
& =T_{k}(t) \\
& -J_{\tau}^{\alpha}\left\{D_{\tau}^{\alpha} T_{k}(\tau)-\left[f(x, y, z) \frac{\partial^{2} T_{k}}{\partial x^{2}}+g(x, y, z) \frac{\partial^{2} T_{k}}{\partial y^{2}}\right.\right. \\
& \left.\left.+h(x, y, z) \frac{\partial^{2} T_{k}}{\partial z^{2}}\right]\right\} .
\end{aligned}
$$

By using the Lemma 11, we finally have

$$
\begin{aligned}
T_{k+1}(t)=\sum_{i=0}^{m-1} T_{k}^{(i)}\left(0^{+}\right) \frac{t^{i}}{i !} & \\
+J_{\tau}^{\alpha}[ & f(x, y, z) \frac{\partial^{2} T_{k}}{\partial x^{2}}+g(x, y, z) \frac{\partial^{2} T_{k}}{\partial y^{2}} \\
& \left.+h(x, y, z) \frac{\partial^{2} T_{k}}{\partial z^{2}}\right] .
\end{aligned}
$$

\section{Applications and Results}

Example 17. Consider the following fractional model for heat conduction in polar bear hairs proposed by Qing-Li et al. [40]:

$$
D_{t}^{\alpha} T+D \frac{\partial^{2} T}{\partial x^{2}}=0, \quad x \in(0,1), t>0,0<\alpha \leq 1,
$$


where $D_{t}^{\alpha} T$ is the modified Rieman-Liouville derivative and $D$ is a constant, with the initial condition

$$
\begin{aligned}
T(x, 0)= & a-\frac{a-b}{1-\exp (-1 / k D)} \\
& +\frac{a-b}{1-\exp (-1 / k D)} \exp \left(-\frac{x}{k D}\right),
\end{aligned}
$$

where $a$ is the body temperature, $b$ is the environment temperature, and $k$ is a constant. When $\alpha=1$, the exact solution of (46) is

$$
\begin{aligned}
T(x, t)= & a-\frac{a-b}{1-\exp (-1 / k D)} \\
& +\frac{a-b}{1-\exp (-1 / k D)} \exp \left(-\frac{x}{k D}-\frac{t}{D k^{2}}\right) .
\end{aligned}
$$

According to the general iteration formula of VIM, one can get the iteration formulation as follows:

$$
T_{n+1}(x, t)=T(x, 0)-J^{\alpha}\left(D \frac{\partial^{2} T_{n}}{\partial x^{2}}\right) .
$$

For the convenience, let $c=(a-b) /(1-\exp (-1 / k D))$ and $d=a-c$, then

$$
T_{0}=d+c \exp \left(-\frac{x}{k D}\right) .
$$

Starting with the initial value as shown in (52), we can derive

$$
\begin{aligned}
T_{1}(x, t) & =d+c \exp \left(-\frac{x}{k D}\right)\left[1-\frac{1}{D k^{2}} \frac{\Gamma(1)}{\Gamma(1+\alpha)} t^{\alpha}\right], \\
T_{2}(x, t)=d & +c \exp \left(-\frac{x}{k D}\right) \\
\times & {\left[1-\frac{1}{D k^{2}} \frac{\Gamma(1)}{\Gamma(1+\alpha)} t^{\alpha}+\frac{1}{\left(D k^{2}\right)^{2}} \frac{\Gamma(1)}{\Gamma(1+2 \alpha)} t^{2 \alpha}\right], }
\end{aligned}
$$

and then

$$
T_{n}(x, t)=d+c \exp \left(-\frac{x}{k D}\right) \sum_{m=0}^{n} \frac{1}{\Gamma(1+m \alpha)}\left(\frac{(-t)^{\alpha}}{D k^{2}}\right)^{m} .
$$

So, the solution is

$$
T(x, t)=\lim _{n \rightarrow \infty} T_{n}(x, t)=d+c \exp \left(-\frac{1}{k D} x\right) E_{\alpha}\left(-\frac{t^{\alpha}}{D k^{2}}\right),
$$

which is the exact solution.

Example 18. Consider the one-dimensional fractional heatlike equation:

$$
\frac{\partial^{\alpha} u}{\partial t^{\alpha}}=\frac{1}{2} x^{2} \frac{\partial^{2} u}{\partial x^{2}}, \quad t>0,0<x<1,1<\alpha \leq 2
$$

subject to the initial condition

$$
u(x, 0)=x, \quad \frac{\partial u(x, 0)}{\partial t}=x^{2},
$$

when $\alpha=2$ and the exact solution is $u(x, t)=x+x^{2} \sinh (t)$.

According to the general iteration formula of VIM, one can get the following formula of iteration:

$$
u_{k+1}(x, t)=u_{k}(x, 0)+\frac{\partial u_{k}(x, 0)}{\partial t} t+J^{\alpha}\left(\frac{1}{2} x^{2} \frac{\partial^{2} u_{k}}{\partial x^{2}}\right) .
$$

Starting with an initial approximation $u_{0}=x+x^{2} t$, one can obtain

$$
\begin{aligned}
& u_{1}=x+x^{2} t+\frac{1}{\Gamma(2+\alpha)} x^{2} t^{1+\alpha} \\
& u_{2}=x+x^{2}\left[t+\frac{t^{1+\alpha}}{\Gamma(2+\alpha)}+\frac{t^{1+2 \alpha}}{\Gamma(2+2 \alpha)}\right], \\
& u_{3}=x+x^{2}\left[t+\frac{t^{1+\alpha}}{\Gamma(2+\alpha)}+\frac{t^{1+2 \alpha}}{\Gamma(2+2 \alpha)}+\frac{t^{1+3 \alpha}}{\Gamma(2+3 \alpha)}\right] .
\end{aligned}
$$

The solution in a series form is given by

$$
\begin{aligned}
u(x, t) & =\lim _{k \rightarrow \infty} u_{k}(x, t) \\
& =x+x^{2} t \sum_{k=0}^{\infty} \frac{t^{k \alpha}}{\Gamma(k \alpha+2)}=x+x^{2} t E_{\alpha, 2}\left(t^{\alpha}\right),
\end{aligned}
$$

where $E_{\alpha, 2}\left(t^{\alpha}\right)$ denotes the two-parameter Mittag-Leffler function. The result obtained in (56) is exactly the same result, obtained by Momani [3] and Faraz et al. [23].

Example 19. Consider the one-dimensional fractional heatlike equation:

$$
\begin{aligned}
& \frac{\partial^{2 \alpha} u}{\partial t^{2 \alpha}}=\frac{1}{12}\left(x^{2} u_{x x}+y^{2} u_{y y}\right), \\
& 0<x, \quad y<1,0.5<\alpha \leq 1, t>0,
\end{aligned}
$$

subject to the boundary conditions

$$
\begin{array}{ll}
u(0, y, t)=0, & u(1, y, t)=4 \cosh t, \\
u(x, 0, t)=0, & u(x, 1, t)=4 \sinh t,
\end{array}
$$

and the initial condition

$$
u(x, y, 0)=x^{4}, \quad u_{t}(x, y, 0)=y^{4} .
$$

The exact solution $\alpha=1$ was found to be [41]

$$
u(x, y, t)=x^{4} \cosh t+y^{4} \sinh t .
$$


According to the general iteration formula of VIM, one can get the following formula of iteration:

$$
u_{k+1}(x, t)=u_{k}(x, t)-J^{2 \alpha}\left(\frac{\partial^{2 \alpha} u}{\partial t^{2 \alpha}}-\frac{1}{12}\left(x^{2} u_{x x}+y^{2} u_{y y}\right)\right) \text {. }
$$

Starting with an initial approximation $u_{0}(x, y, t)=$ $u(x, y, 0)=x^{4}+y^{4} t$, one can obtain the following successive approximate:

$$
\begin{aligned}
u_{1}= & x^{4}\left[1+\frac{1}{\Gamma(1+2 \alpha)} t^{2 \alpha}\right]+y^{4} t\left[1+\frac{1}{\Gamma(2+2 \alpha)} t^{2 \alpha}\right] \\
u_{2}= & x^{4}\left[1+\frac{1}{\Gamma(1+2 \alpha)} t^{2 \alpha}+\frac{1}{\Gamma(1+4 \alpha)} t^{4 \alpha}\right] \\
& +y^{4} t\left[1+\frac{1}{\Gamma(2+2 \alpha)} t^{2 \alpha}+\frac{1}{\Gamma(2+4 \alpha)} t^{4 \alpha}\right] \\
u_{3}= & x^{4}\left[1+\frac{1}{\Gamma(1+2 \alpha)} t^{2 \alpha}+\frac{1}{\Gamma(1+4 \alpha)} t^{4 \alpha}+\frac{1}{\Gamma(1+6 \alpha)} t^{6 \alpha}\right] \\
+ & y^{4} t\left[1+\frac{1}{\Gamma(2+2 \alpha)} t^{2 \alpha}+\frac{1}{\Gamma(2+4 \alpha)} t^{4 \alpha}\right. \\
& \left.+\frac{1}{\Gamma(1+6 \alpha)} t^{6 \alpha}\right] .
\end{aligned}
$$

The solution in a series form is given by

$$
\begin{aligned}
u(x, t) & =\lim _{k \rightarrow \infty} u_{k}(x, t) \\
& =x^{4} \sum_{k=0}^{\infty} \frac{t^{2 k \alpha}}{\Gamma(2 k \alpha+1)}+y^{4} t \sum_{k=0}^{\infty} \frac{t^{2 k \alpha}}{\Gamma(2 k \alpha+2)} \\
& =x^{4} t E_{2 \alpha, 1}\left(t^{2 \alpha}\right)+y^{4} t E_{2 \alpha, 2}\left(t^{2 \alpha}\right),
\end{aligned}
$$

where $E_{2 \alpha, 2}\left(t^{\alpha}\right)$ denotes the two-parameter Mittag-Leffler function.

Example 20. Consider the one-dimensional fractional heatlike equation:

$$
\begin{array}{r}
\frac{\partial^{2 \alpha} u}{\partial t^{2 \alpha}}=x^{2} y^{2} z^{2}+\frac{1}{2}\left(x^{2} u_{x x}+y^{2} u_{y y}+z^{2} u_{z z}\right), \\
0<x, y, z<1, \quad 0.5<\alpha \leq 1, \quad t>0,
\end{array}
$$

subject to the boundary conditions

$$
\begin{gathered}
u(0, y, z, t)=y^{2}\left(e^{t}-1\right)+z^{2}\left(e^{-t}-1\right), \\
u(1, y, z, t)=\left(1+y^{2}\right)\left(e^{t}-1\right)+z^{2}\left(e^{-t}-1\right), \\
u(x, 0, z, t)=x^{2}\left(e^{t}-1\right)+z^{2}\left(e^{-t}-1\right), \\
u(x, 1, z, t)=\left(1+x^{2}\right)\left(e^{t}-1\right)+z^{2}\left(e^{-t}-1\right), \\
u(x, y, 0, t)=\left(x^{2}+y^{2}\right)\left(e^{t}-1\right), \\
u(x, y, 1, t)=\left(x^{2}+y^{2}\right)\left(e^{t}-1\right)+\left(e^{-t}-1\right),
\end{gathered}
$$

and the initial condition

$$
u(x, y, z, 0)=0, \quad u_{t}(x, y, z, 0)=x^{2}+y^{2}-z^{2} .
$$

The exact solution $\alpha=1$ was found to be [41]

$$
u(x, y, t)=\left(x^{2}+y^{2}\right) e^{t}+z^{2} e^{-t}-\left(x^{2}+y^{2}+z^{2}\right)
$$

According to the general iteration formula of VIM, one can get the following formula of iteration:

$$
\begin{aligned}
u_{k+1} & (x, t) \\
= & u_{k}(x, t) \\
& -J^{2 \alpha}\left(\frac{\partial^{2 \alpha} u}{\partial t^{2 \alpha}}-x^{2} y^{2} z^{2}-\frac{1}{2}\left(x^{2} u_{x x}+y^{2} u_{y y}+z^{2} u_{z z}\right)\right)
\end{aligned}
$$

Starting with an initial approximation,

$$
\begin{aligned}
u_{0}(x, y, z, t)= & \left(x^{2}+y^{2}\right)\left(t+\frac{t^{2 \alpha}}{\Gamma(2 \alpha+1)}\right) \\
& +z^{2}\left(-t+\frac{t^{2 \alpha}}{\Gamma(2 \alpha+1)}\right),
\end{aligned}
$$

which was given by [41]. One can obtain the following successive approximate:

$$
\begin{aligned}
u_{1}= & \left(x^{2}+y^{2}\right) \\
& \times\left[1+t+\frac{t^{2 \alpha}}{\Gamma(1+2 \alpha)}+\frac{t^{1+2 \alpha}}{\Gamma(2+2 \alpha)}+\frac{t^{4 \alpha}}{\Gamma(1+4 \alpha)}\right] \\
& +z^{2}\left[1-t+\frac{t^{2 \alpha}}{\Gamma(1+2 \alpha)}-\frac{t^{1+2 \alpha}}{\Gamma(2+2 \alpha)}+\frac{t^{4 \alpha}}{\Gamma(1+4 \alpha)}\right] \\
& -x^{2} y^{2} z^{2}, \\
u_{2}= & \left.x^{2}+y^{2}\right)\left[1+t+\frac{t^{2 \alpha}}{\Gamma(1+2 \alpha)}+\frac{t^{1+2 \alpha}}{\Gamma(2+2 \alpha)}\right. \\
& +z^{2}\left[1-t+\frac{t^{4 \alpha}}{\Gamma(1+4 \alpha)}+\frac{t^{1+4 \alpha}}{\Gamma(2+4 \alpha)}+\frac{t^{6 \alpha}}{\Gamma(1+6 \alpha)}\right] \\
-\frac{t^{1+4 \alpha}}{\Gamma(2+4 \alpha)}+\frac{t^{1+2 \alpha}}{\Gamma(1+6 \alpha)}+\frac{t^{4 \alpha}}{\Gamma(2+2 \alpha)} & \frac{t^{6 \alpha}}{\Gamma(1+4 \alpha)} \\
& {\left[x^{2} y^{2} z^{2},\right.}
\end{aligned}
$$

when $\alpha=1$ and the exact solution for (68) is

$$
u(x, y, t)=\left(x^{2}+y^{2}\right) e^{t}+z^{2} e^{-t}-\left(x^{2}+y^{2}+z^{2}\right) .
$$

From above procedure of solution, one can conclude that the result obtained by the fractional variational iteration method (FVIM) is the same as the decomposition method [3]. 


\section{Conclusion}

VIM has been known as a powerful tool for solving many fractional differential equations. In this paper, we derive a general iteration formula of VIM for fractional heat- and wave-like equations with variable coefficients. There are two points to make here. First, the Lagrange multiplier of the method is identified in a more accurate way by employing the Laplace transform. Second, our iteration formula still holds true in the case of $\alpha>1$. All the examples show that the results of the proposed method are more accurate than those obtained by the classical VIM and the same as the ADM but not require the calculation of Adomian's polynomials.

\section{Acknowledgments}

This work is supported by National Natural Science Foundation of China (Grant no. 41105063). The authors are very grateful to reviewers for carefully reading the paper and for their comments and suggestions which have improved the paper.

\section{References}

[1] Z. Odibat and S. Momani, "Numerical methods for nonlinear partial differential equations of fractional order," Applied Mathematical Modelling, vol. 32, no. 1, pp. 28-39, 2008.

[2] S. Momani and Z. Odibat, "Analytical approach to linear fractional partial differential equations arising in fluid mechanics," Physics Letters A, vol. 355, no. 4-5, pp. 271-279, 2006.

[3] S. Momani, "Analytical approximate solution for fractional heat-like and wave-like equations with variable coefficients using the decomposition method," Applied Mathematics and Computation, vol. 165, no. 2, pp. 459-472, 2005.

[4] S. Momani and Z. Odibat, "Analytical solution of a timefractional Navier-Stokes equation by Adomian decomposition method," Applied Mathematics and Computation, vol. 177, no. 2, pp. 488-494, 2006.

[5] G.-C. Wu and J.-H. He, "Fractional adomian decomposition method," http://arxiv.org/abs/1006.5264.

[6] S. Abbasbandy, "Numerical solutions of the integral equations: homotopy perturbation method and Adomian's decomposition method," Applied Mathematics and Computation, vol. 173, no. 1, pp. 493-500, 2006.

[7] I.-C. Liu and A. M. Megahed, "Homotopy perturbation method for thin film flow and heat transfer over an unsteady stretching sheet with internal heating and variable heat flux," Journal of Applied Mathematics, vol. 2012, Article ID 418527, 12 pages, 2012.

[8] S. H. Hosseinnia, A. Ranjbar, and S. Momani, "Using an enhanced homotopy perturbation method in fractional differential equations via deforming the linear part," Computers \& Mathematics with Applications, vol. 56, no. 12, pp. 3138-3149, 2008.

[9] S. Abbasbandy, "Homotopy perturbation method for quadratic Riccati differential equation and comparison with Adomian's decomposition method," Applied Mathematics and Computation, vol. 172, no. 1, pp. 485-490, 2006.

[10] S. Abbasbandy, "Modified homotopy perturbation method for nonlinear equations and comparison with Adomian decomposition method," Applied Mathematics and Computation, vol. 172, no. 1, pp. 431-438, 2006.
[11] J. H. He, "Variational iteration method-a kind of non-linear analytical technique: some examples," International Journal of Non-Linear Mechanics, vol. 34, no. 4, pp. 699-708, 1999.

[12] J.-H. He, "Variational iteration method for autonomous ordinary differential systems," Applied Mathematics and Computation, vol. 114, no. 2-3, pp. 115-123, 2000.

[13] J.-H. He, "Approximate analytical solution for seepage flow with fractional derivatives in porous media," Computer Methods in Applied Mechanics and Engineering, vol. 167, no. 1-2, pp. 57-68, 1998.

[14] J. H. He, "Asymptotic methods for solitary solutions and compactons," Abstract and Applied Analysis, vol. 2012, Article ID 916793, 130 pages, 2012.

[15] J. H. He, "Some applications of nonlinear fractional differential equations and their approximations," Bulletin of Science and Technology, vol. 15, no. 2, pp. 86-90, 1999.

[16] T. A. Nofal, "Approximate solutions for nonlinear initial value problems using the modified variational iteration method," Journal of Applied Mathematics, vol. 2012, Article ID 370843, 19 pages, 2012.

[17] Y. M. R, M. S. M. Noorani, and I. Hashim, "Variational iteration method for fractional heat- and wave-like equations," Nonlinear Analysis: Real World Applications, vol. 10, no. 3, pp. 1854-1869, 2009.

[18] Z. M. Odibat and S. Momani, "Application of variational iteration method to nonlinear differential equations of fractional order," International Journal of Nonlinear Sciences and Numerical Simulation, vol. 7, no. 1, pp. 27-34, 2006.

[19] G. E. Drăgănescu, "Application of a variational iteration method to linear and nonlinear viscoelastic models with fractional derivatives," Journal of Mathematical Physics, vol. 47, no. 8, Article ID 082902, 9 pages, 2006.

[20] D. D. Ganji and S. H. Hashemi Kachapi, "Analysis of nonlinear equations in fluids," Progress in Nonlinear Science, vol. 2, pp. 1293, 2011.

[21] D. D. Ganji and S. H. Hashemi Kachapi, "Analytical and numerical methods in engineering and applied sciences," Progress in Nonlinear Science, vol. 3, pp. 1-579, 2011.

[22] G.-C. Wu, "Variational iteration method for $q$-difference equations of second order," Journal of Applied Mathematics, vol. 2012, Article ID 102850, 5 pages, 2012.

[23] N. Faraz, Y. Khan, H. Jafari, A. Yildirim, and M. Madani, "Fractional variational iteration method via modified RiemannLiouville derivative," Journal of King Saud University-Science, vol. 23, no. 4, pp. 413-417, 2011.

[24] E. J. Ali, "Modified treatment of initial boundary value problems for one dimensional heat-like and wave-like equations using variational iteration method," Applied Mathematical Sciences, vol. 6, no. 33-36, pp. 1613-1626, 2012.

[25] G.-C. Wu and E. W. M. Lee, "Fractional variational iteration method and its application," Physics Letters A, vol. 374, no. 25, pp. 2506-2509, 2010.

[26] G.-C. Wu, "A fractional variational iteration method for solving fractional nonlinear differential equations," Computers \& Mathematics with Applications, vol. 61, no. 8, pp. 2186-2190, 2011.

[27] Z. Odibat and S. Momani, "The variational iteration method: an efficient scheme for handling fractional partial differential equations in fluid mechanics," Computers \& Mathematics with Applications, vol. 58, no. 11-12, pp. 2199-2208, 2009.

[28] S. Liao, "On the homotopy analysis method for nonlinear problems," Applied Mathematics and Computation, vol. 147, no. 2, pp. 499-513, 2004. 
[29] S. Abbasbandy and A. Shirzadi, "Homotopy analysis method for multiple solutions of the fractional Sturm-Liouville problems," Numerical Algorithms, vol. 54, no. 4, pp. 521-532, 2010.

[30] I. Podlubny, Fractional Differential Equations: An Introduction To Fractional Derivatives, Fractional Differential Equations, To Methods of Their Solution and Some of Their Applications, vol. 198 of Mathematics in Science and Engineering, Academic Press, San Diego, Calif, USA, 1998.

[31] G. Jumarie, "Stochastic differential equations with fractional Brownian motion input," International Journal of Systems Science, vol. 24, no. 6, pp. 1113-1131, 1993.

[32] G. Jumarie, "On the representation of fractional Brownian motion as an integral with respect to $(\mathrm{d} t)^{a}$," Applied Mathematics Letters, vol. 18, no. 7, pp. 739-748, 2005.

[33] G. Jumarie, "On the solution of the stochastic differential equation of exponential growth driven by fractional Brownian motion," Applied Mathematics Letters, vol. 18, no. 7, pp. 817-826, 2005.

[34] G. Jumarie, "Modified Riemann-Liouville derivative and fractional Taylor series of nondifferentiable functions further results," Computers \& Mathematics with Applications, vol. 51, no. 9-10, pp. 1367-1376, 2006.

[35] G. Jumarie, "New stochastic fractional models for Malthusian growth, the Poissonian birth process and optimal management of populations," Mathematical and Computer Modelling, vol. 44, no. 3-4, pp. 231-254, 2006.

[36] G. Jumarie, "Stock exchange fractional dynamics defined as fractional exponential growth driven by (usual) Gaussian white noise. Application to fractional Black-Scholes equations," Insurance Mathematics \& Economics, vol. 42, no. 1, pp. 271-287, 2008.

[37] G. Jumarie, "Table of some basic fractional calculus formulae derived from a modified Riemann-Liouville derivative for nondifferentiable functions," Applied Mathematics Letters, vol. 22, no. 3, pp. 378-385, 2009.

[38] G. Jumarie, "Laplace's transform of fractional order via the Mittag-Leffler function and modified Riemann-Liouville derivative," Applied Mathematics Letters, vol. 22, no. 11, pp. 16591664, 2009.

[39] L. Kexue and P. Jigen, "Laplace transform and fractional differential equations," Applied Mathematics Letters, vol. 24, no. 12, pp. 2019-2023, 2011.

[40] W. Qing-Li, J. H. He, and L. Zheng-Biao, "Fractional model for heat conduction in polar bear hairs," Thermal Science, vol. 16, no. 2, pp. 339-342, 2012.

[41] D.-H. Shou and J.-H. He, "Beyond Adomian method: the variational iteration method for solving heat-like and wave-like equations with variable coefficients," Physics Letters A, vol. 372, no. 3, pp. 233-237, 2008. 


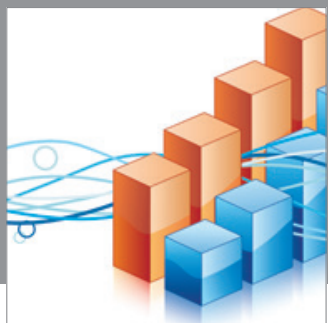

Advances in

Operations Research

mansans

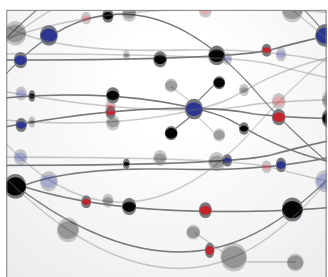

The Scientific World Journal
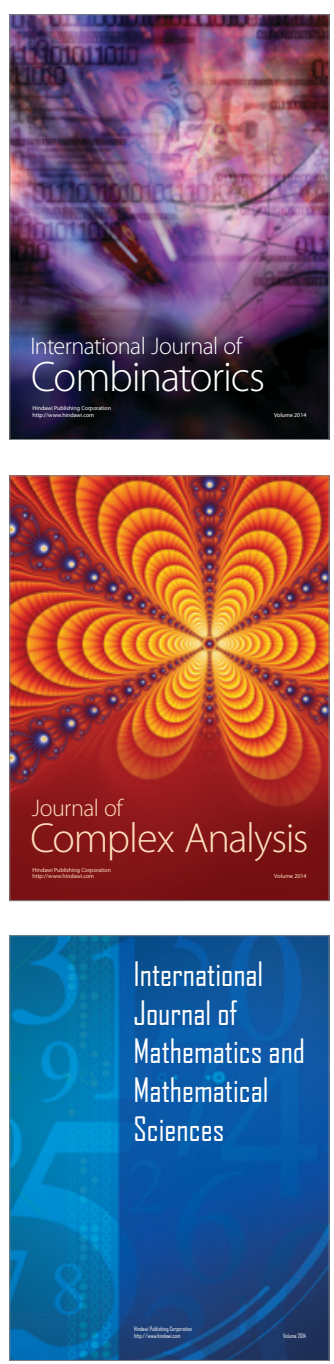
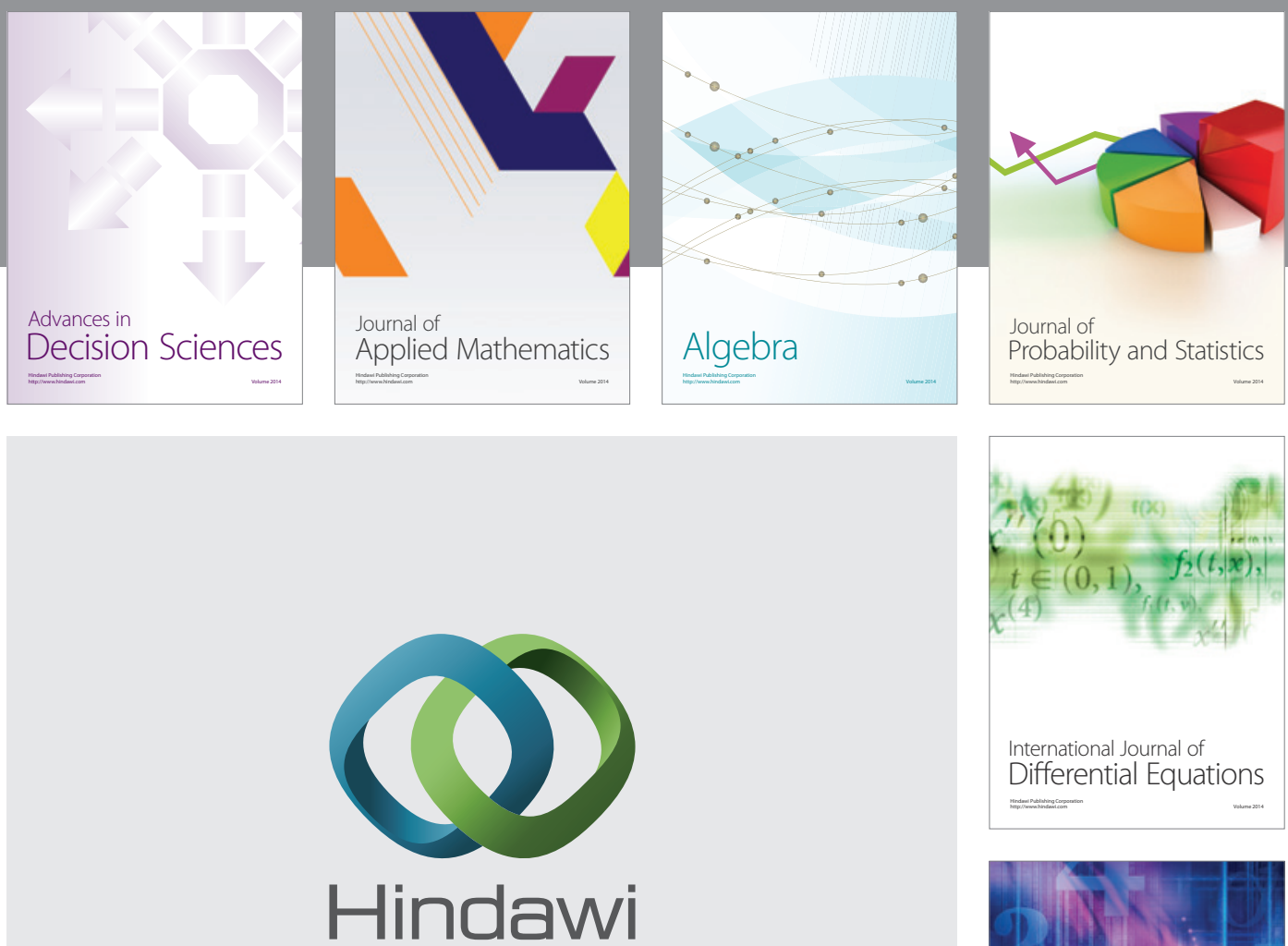

Submit your manuscripts at http://www.hindawi.com
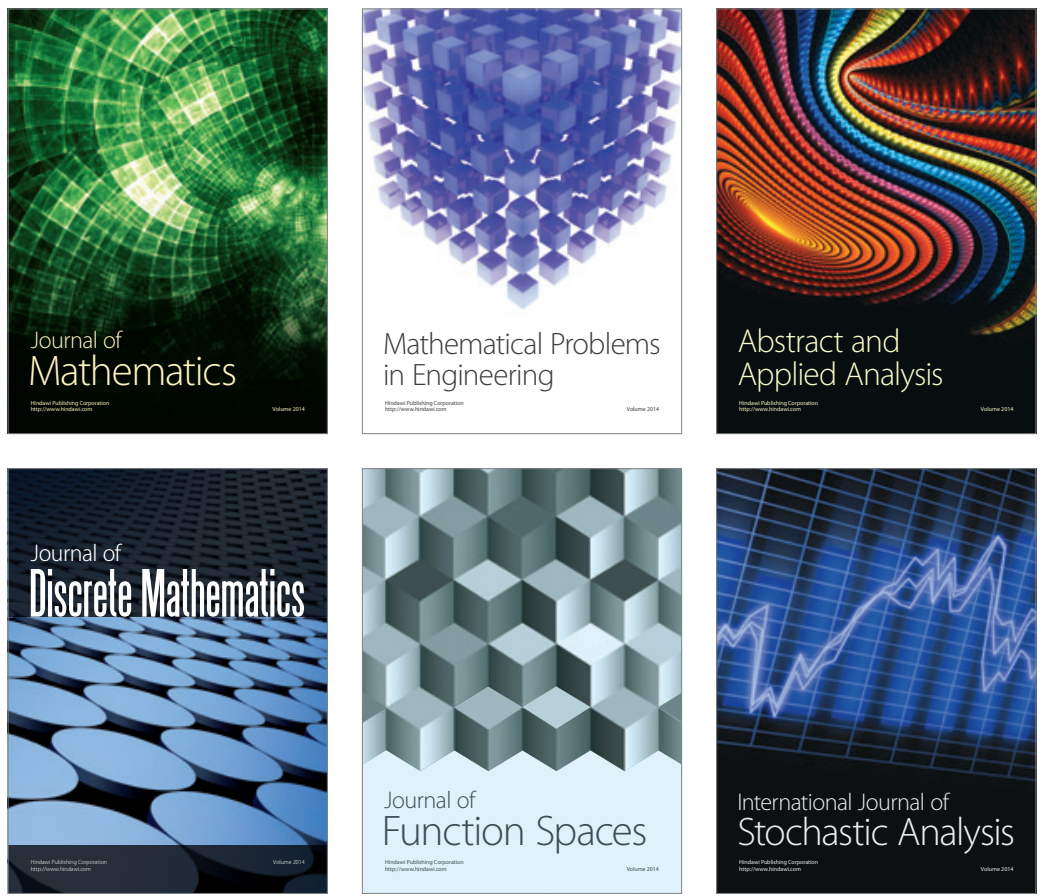

Journal of

Function Spaces

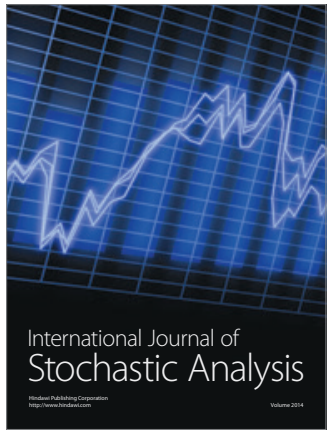

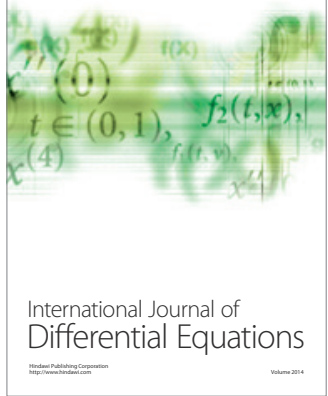
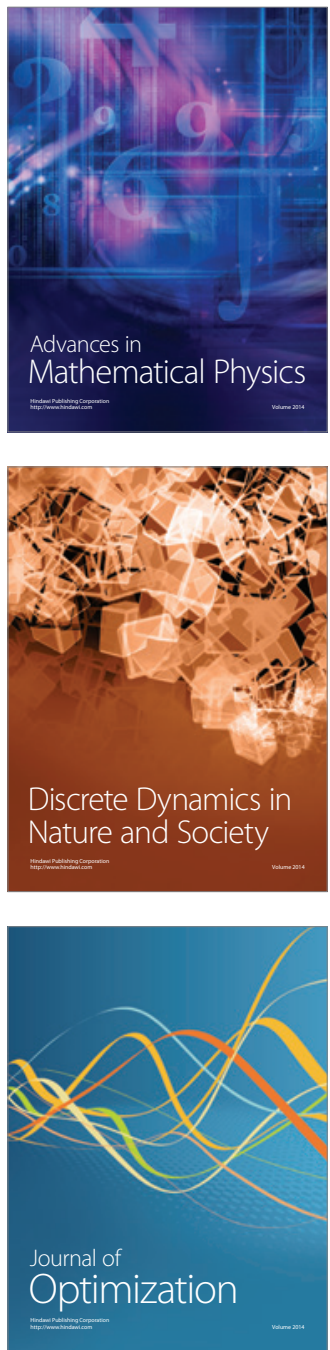